\title{
Habilidades motrices y su relación con las actividades y creencias parentales en preescolares; comparaciones por nivel socio-económico
}

\section{Preschoolers' motor skills and their relation to parental activities and beliefs: comparisons by socio-economic status}

Dra. Carolina Jiménez Liraํㅜ Dra. Elia Verónica Benavides Pandoํㅜ Dra. Martha Ornelas Contreras $^{1}$; Dra. Judith Margarita Rodríguez-Villalobos ${ }^{1}$; Dra. Susana Olga Lira Guerra ${ }^{2}$; M.P.P. Azaneth Laguna Celia ${ }^{1}$ Contacto: Elia Verónica Benavides ebenavides@uach.mx

${ }^{1}$ Universidad Autónoma de Chihuahua, Facultad de Ciencias de la Cultura Física

${ }^{2}$ Servicios de Salud del Estado de Chihuahua 


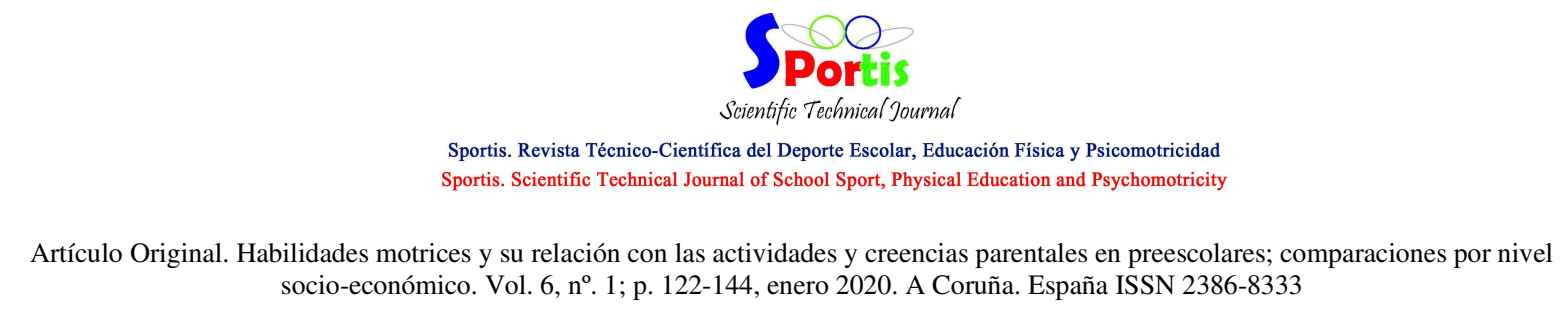

\title{
Resumen
}

El objetivo de esta investigación es analizar cómo se relacionan las habilidades motrices gruesas y finas en preescolares, la frecuencia de actividades motrices reportadas por los padres y las creencias sobre el desarrollo motor en diferente Nivel SocioEconómico (NSE). Participaron 75 padres de familia y sus respectivos hijos de NSE bajo y alto. La frecuencia con que realizan actividades motrices con sus hijos y sus creencias sobre el desarrollo motor se registraron por medio de un cuestionario; las habilidades motrices se evaluaron con las sub-escales de motricidad fina y gruesa del Inventario de Desarrollo Battelle (BDI-2). En los resultados los padres afirmaron otorgar mayor importancia al desarrollo de la motricidad fina que al desarrollo de la motricidad gruesa. Los niños de NSE bajo obtuvieron una puntuación más alta en motricidad gruesa que sus pares de NSE alto, sin embargo no se encontraron diferencias entre las puntuaciones de motricidad fina entre ambos grupos. Concluimos que el desarrollo de las habilidades motrices en preescolar no parece estar asociado a las creencias de los padres ni a la frecuencia con que suelen realizar actividades motrices con sus hijos; sin embargo existen diferencias por NSE en el desempeño de los menores.

\section{Palabras Clave}

Desarrollo motor; preescolar; psicomotricidad; creencias parentales; nivel socioeconómico.

\begin{abstract}
The purpose of the present study is to analyze the relationship between preschoolers' gross and fine motor skills, parent reported frequency of motor activities, and parental beliefs about motor development in different socio-economic contexts. Seventy-five parents and their children from low and high socio-economic status participated in the study. The frequency with which parents engaged in motor activities with their children and their beliefs about motor development were assessed using a questionnaire; children's motor skills were assessed using the fine and gross motor skill subscales from the Battelle Developmental Inventory (BDI-2). Results show that parents report that they consider the development of fine- as more important than gross motor skills. Children from Low SES performed better on the gross motor skill assessment than their higher SES counterparts, however, performance did not differ by SES on the fine motor skill assessment. We conclude that the development of motor skills in preschool does not appear to be associated to parental beliefs or to parent reported frequency of motor activities; however, we did find differences by SES on children's performance.
\end{abstract}

\section{Keywords}

Motor development; preschool; psychomotricity; parental beliefs; socio-economic status.

Para citar este artículo utilice la siguiente referencia: Jiménez, C.; Benavides, E. V.; Ornelas, M.; Rodríguez, J.M.; Lira, S.O.; Laguna, A. (2020). Habilidades motrices y su relación con las actividades y creencias parentales en preescolares; comparaciones por nivel socio-económico. Sportis Sci J, 6 (1), 122144. DOI: https://doi.org/10.17979/sportis.2020.6.1.5388 http://revistas.udc.es/ 
Artículo Original. Habilidades motrices y su relación con las actividades y creencias parentales en preescolares; comparaciones por nivel socio-económico. Vol. 6, no. 1; p. 122-144, enero 2020. A Coruña. España ISSN 2386-8333
\end{abstract}

\title{
Introducción
}

La motricidad es un eje fundamental del desarrollo humano. A través de ella, el niño conoce su cuerpo, sus capacidades, sus límites; explora su entorno y le permite realizar hazañas que van desde patear una pelota hasta pintar una obra de arte. Durante la etapa preescolar, el movimiento y el juego propician tanto el desarrollo de las capacidades físicas como de aprendizajes en otras áreas tales como: lenguaje y vocabulario, conocimiento del medio, que fortalecen la autonomía personal (Arufe-Giráldez, 2020). El desarrollo motor en edades tempranas se da en el ámbito del hogar, siendo los padres los responsables de proporcionar al niño la estimulación necesaria para que alcance su máximo potencial motor (Freitas, Gabbard, Caçola, Montebelo y Santos, 2013). Al referirse a las habilidades motoras básicas que deben adquirir los niños en las primeras edades, Castillo (2013) indica que estas deben ser: atrapar, correr, lanzar, saltar, batear una pelota, correr a gran velocidad y saltar una cuerda, puntualiza que estas habilidades son las que sientan las bases de acciones de movimientos concretos que los benefician. En la primera infancia se deben adquirir estos patrones motores básicos, con que se pueden construir nuevas opciones de movimiento y desarrollar correctamente las capacidades motrices y las habilidades básicas, se persigue aprovechar el periodo sensitivo, con particularidades que no se repiten en ninguna otra etapa de la vida. En el desarrollo físico de las niñas y de los niños están involucrados el movimiento y la locomoción, la estabilidad y el equilibrio, la manipulación, la proyección y la recepción como capacidades motrices (Miranda, Jeffers, Duarte, y Rey, 2019). Luego, es en esta etapa, cuando los niños desarrollan su motricidad gruesa, la cual se refiere a movimientos amplios que involucran los músculos de todo el cuerpo y la motricidad fina que implica movimientos coordinados de la mano (Berruezo, 2000). El desarrollo psicomotor se refiere a la adquisición de destrezas corporales mediante interacciones intrínsecas y extrínsecas que se observan en el niño y niña durante toda la infancia y que lo convertirán en un sujeto con poder locomotrizmanipulativo. Lo anterior con un fin: la ganancia de autonomía y capacidad de interactuar con el mundo y poder transformarlo (Osorio, Cortés, Herrera y Orozco, 2017).

Existen diversos factores que se relacionan con las habilidades motrices en edad

Para citar este artículo utilice la siguiente referencia: Jiménez, C.; Benavides, E. V.; Ornelas, M.; Rodríguez, J.M.; Lira, S.O.; Laguna, A. (2020). Habilidades motrices y su relación con las actividades y creencias parentales en preescolares; comparaciones por nivel socio-económico. Sportis Sci J, 6 (1), 122144. DOI: https://doi.org/10.17979/sportis.2020.6.1.5388 
Artículo Original. Habilidades motrices y su relación con las actividades y creencias parentales en preescolares; comparaciones por nivel socio-económico. Vol. 6, nº. 1; p. 122-144, enero 2020. A Coruña. España ISSN 2386-8333

preescolar tales como a) la frecuencia con que los padres practican actividades motrices en casa (Rodrigues, Raraiva y Gabbard, 2005), b) las creencias con respecto a la importancia de los hitos del desarrollo motor, y c) el nivel socio-económico (Bustamante, Caballero Cartagena, Enciso Sarria, Salazar Távara, Teixeira e Seabra, Garganta de Silva, y Ribeiro Maia, 2008; Liu, Hoffman, y Hamilton, 2015; Rizzoli-Córdoba et al., 2015).
\end{abstract}

Se ha hipotetizado que durante la primera infancia, el ambiente educativo en el hogar y las actividades con que los padres estimulan el desarrollo motor de sus hijos tienen un efecto directo sobre las habilidades motrices (Rodrigues, et al., 2005), sin embargo se han encontrado hallazgos contradictorios; por ejemplo, Abbott, Bartett, Kneale Fanning, y Kramer, (2000) analizaron la relación entre medidas globales de ambiente en el hogar (p. ej. responsividad materna, aceptación, organización, material didáctico, etc.) y el desarrollo motor de menores de 5 a 8 meses sin encontrar relaciones significativas; por su parte, Miquelote, Santos, Caçola, Montebelo y Gabbard, (2012) encontraron una relación predictiva entre aspectos del ambiente en el hogar tales como reportes de actividades diarias y disponibilidad de materiales de juego con medidas de motricidad en niños de 9 a 15 meses. En México, Osorio, Torres-Sánchez, Hernández, López Carrillo, y Schnaas (2010) midieron aspectos de la estimulación tales como interacción madre-hijo, reforzamiento positivo, y establecimiento de límites en padres de niños de 36 meses y su relación con las habilidades motrices gruesa y fina en sus hijos; se encontró una relación positiva entre la estimulación en el hogar y el desempeño motor de los menores. Se debe señalar que estos estudios no indagaron específicamente sobre actividades motrices llevadas a cabo por los padres sino que operacionalizaron la estimulación en el hogar con los resultados de un instrumento (HOME) que evalúa aspectos generales de la misma, por lo cual queda la duda sobre qué tipo de actividades motrices realizan los padres en el hogar, la frecuencia y si estas actividades tienen un impacto sobre las habilidades de los menores.

Es de gran interés considerar el contexto del niño, la sociedad tiende a formar a sus integrantes desde diferentes perspectivas, familiar, cultural, comunitario, institucional lo cual posteriormente se ve reflejado en sus valores, ideología, actitudes y demandas, esto a su vez se puede traducir en oportunidades de desenvolvimiento, es decir, de acuerdo al

Para citar este artículo utilice la siguiente referencia: Jiménez, C.; Benavides, E. V.; Ornelas, M.; Rodríguez, J.M.; Lira, S.O.; Laguna, A. (2020). Habilidades motrices y su relación con las actividades y creencias parentales en preescolares; comparaciones por nivel socio-económico. Sportis Sci J, 6 (1), 122144. DOI: https://doi.org/10.17979/sportis.2020.6.1.5388 

socio-económico. Vol. 6, nº. 1; p. 122-144, enero 2020. A Coruña. España ISSN 2386-8333

ambiente en el que se desarrolle el niño tendrá diferentes oportunidades para adquirir experiencias las cuales se convertirán en aprendizajes los que a su vez contribuirán a potenciar sus habilidades. Todo aquello que la sociedad y la familia crean relevante para el aprendizaje del niño será la base para establecer las pautas de del sujeto, por lo tanto, el aprendizaje es un punto clave para su desarrollo integral (Zapata y Restrepo, 2013).

Se ha encontrado además que las actividades realizadas por los padres con sus hijos tales como animarlo a correr (motricidad gruesa) o jugar con plastilina (motricidad fina) así como la frecuencia con que se realizan, están relacionadas con las creencias y expectativas con respecto a la importancia de los hitos del desarrollo motor en padres de menores de entre 1 y 2 años (Gomes, Fonseca, Vinolas Prat, de Castro Magalhães, y de Souza Morais, 2017) y hasta los 66 meses (Silva, Flôres, Corrêa, Cordovil y Copetti, 2017). Estos estudios, sin embargo, no incluyeron medidas de habilidades motrices en los hijos de los participantes, por lo cual es importante contribuir con información que permita analizar la relación entre actividades y creencias paternas, y su relación con las habilidades motrices de los hijos.

Diversos estudios consideran que el nivel socio-económico influye en el desarrollo motor de los niños en edades tempranas. Por ejemplo, Díaz, Bacallao, Vargas-Machuca y Aguilar (2017) afirman que los niños de áreas rurales, hijos de madres con baja escolaridad y pertenecientes a hogares con necesidades básicas insatisfechas exhiben valores más bajos en el desarrollo de las áreas motriz y de lenguaje. El retraso se incrementa al aumentar el número de condiciones de riesgo. En Estados Unidos, Liu et al., (2015) encontraron que niños de nivel socio-económico bajo presentaron puntuaciones significativamente más bajas que sus compañeros de nivel socio-económico más aventajado en pruebas de coordinación viso-motriz y locomoción; en México, Rizzoli-Córdoba et al., (2015) encontraron que el porcentaje de niños menores de 5 años con rezago en el desarrollo de la motricidad gruesa tiende a disminuir con la edad; mientras que el porcentaje de niños con rezago en el desarrollo de la motricidad fina tiende a aumentar con la edad en menores beneficiarios de programas de asistencia social en salud (PROSPERA) quienes en su mayoría son de NSE bajo.

Para citar este artículo utilice la siguiente referencia: Jiménez, C.; Benavides, E. V.; Ornelas, M.; Rodríguez, J.M.; Lira, S.O.; Laguna, A. (2020). Habilidades motrices y su relación con las actividades y creencias parentales en preescolares; comparaciones por nivel socio-económico. Sportis Sci J, 6 (1), 122144. DOI: https://doi.org/10.17979/sportis.2020.6.1.5388 


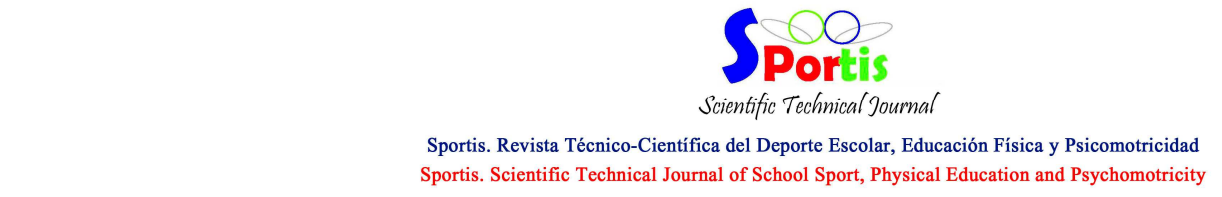

Artículo Original. Habilidades motrices y su relación con las actividades y creencias parentales en preescolares; comparaciones por nivel socio-económico. Vol. 6, nº. 1; p. 122-144, enero 2020. A Coruña. España ISSN 2386-8333

Algunos estudios analizan las diferencias entre las actividades motrices en el hogar, la frecuencia con que se realizan y las creencias de los padres pertenecientes a distintos niveles socio-económicos (p. ej. Leiva y Valdés, 2016). Estas diferencias se relacionan con factores como la educación y conocimiento de los padres con respecto a los hitos del desarrollo motor (Rodríguez-Garcés y Muñoz-Soto, 2017) y el acceso a materiales de estimulación motriz (p. ej., aros, pelotas, suscripción a clubes deportivos) (Miquelote, et al., 2012; Recart y Mathiesen, 2002). Sin embargo, Freitas et al., (2011) encontraron que, mientras que la disponibilidad de espacio y accesorios para promover el desarrollo motor de los niños se relaciona con el nivel socio-económico, las actividades realizadas por los padres no tenían relación con este factor y más bien se relacionaban con la edad de los menores. Los resultados de estos estudios pudieran sugerir que los niños pertenecientes a niveles socio-económicos bajos pudiesen estar en desventaja con respecto a sus pares pertenecientes a familias con mayor poder adquisitivo en cuanto a las oportunidades de utilizar materiales que promuevan la actividad física y el desarrollo motor (Bustamante et al., 2008), pero brindan evidencia de que independientemente del nivel socio-económico los padres realizan actividades motrices que favorecen estas habilidades.

El presente estudio tiene la finalidad de analizar: a) si existe diferencia entre la frecuencia de actividades de motricidad gruesa y fina que reportan los padres de NSE alto y bajo; b) si existe diferencia entre las creencias de los padres de NSE alto y bajo con respecto al desarrollo de las habilidades motrices gruesa y fina; c) si existe diferencia entre las habilidades de motricidad gruesa, y motricidad fina entre preescolares de NSE alto y bajo; d) si las creencias y frecuencia de actividades motrices reportada por los padres se relacionan con las habilidades motrices gruesa y fina obtenidas por sus hijos.

\section{Método}

Todos los procedimientos del presente estudio fueron aprobados por el Comité de Ética en Investigación de la Facultad de Medicina de la Universidad Autónoma de Chihuahua.

\section{Participantes}

Participaron 75 niños que cursan la educación pre-escolar en la ciudad de Chihuahua; 39 pertenecen a dos instituciones públicas (17 mujeres, 22 hombres; $M$ edad =

Para citar este artículo utilice la siguiente referencia: Jiménez, C.; Benavides, E. V.; Ornelas, M.; Rodríguez, J.M.; Lira, S.O.; Laguna, A. (2020). Habilidades motrices y su relación con las actividades y creencias parentales en preescolares; comparaciones por nivel socio-económico. Sportis Sci J, 6 (1), 122144. DOI: https://doi.org/10.17979/sportis.2020.6.1.5388 

socio-económico. Vol. 6, nº. 1; p. 122-144, enero 2020. A Coruña. España ISSN 2386-8333

52.18 meses, $S D=6.94$; rango $=36-66$ meses $)$ y 36 a tres escuelas particulares $(16$ mujeres, 20 hombres; $M$ edad $=51.05$ meses, $S D=9.70$; rango $=37-70$ meses ).

\section{Procedimiento}

Antes de invitar a las instituciones a participar, se solicitó autorización a Servicios Educativos del Estado de Chihuahua, una vez aprobada, se envió un escrito a los directivos de los jardines de niños; en el momento que aceptaron participar se entregaron los consentimientos informados y cuestionarios a los padres de familia quienes los regresaban a la maestra de sus hijos en un sobre sellado para proteger la confidencialidad de los datos. Únicamente se evaluaron aquellos niños que voluntariamente aceptaron participar y que contaban con la autorización de sus padres o tutores.

\section{Instrumentos de medición}

Este estudio forma parte de un macro-proyecto en el cual los niños completaran 10 pruebas de lecto-escritura y matemáticas además de las 2 sub-escalas de motricidad fina y gruesa. El tiempo requerido para completar la batería de pruebas fue de aproximadamente una hora repartida en 2 sesiones llevadas a cabo en distintos días dentro de una misma semana.

Sub-escalas de motricidad gruesa y fina del Inventario de Desarrollo Battelle. Se evaluaron habilidades motrices con las sub-escalas de motricidad fina y gruesa del Inventario de Desarrollo Battelle Segunda Edición BDI-2. El Inventario de Desarrollo Battelle es una prueba estandarizada que se puede aplicar a niños de 0 a 8 años de edad. El instrumento tiene índices de fiabilidad mayores a .85 en todas las sub-escalas que ha sido utilizada en estudios que investigan en desarrollo de menores.

Las sub-escalas de motricidad gruesa y motricidad fina son adecuadas para evaluar a niños de 0 a 6 años. La sub-escala de motricidad gruesa consta en total de 45 ítems que incluyen, por ejemplo, pedirle al niño que lance una pelota, que salte hacia delante en un solo pie, que suba y baje escaleras. La sub-escala de motricidad fina consta de 30 ítems entre los cuales se le solicita al niño por ejemplo que recorte con tijeras, ate un nudo, que engarce unas cuentas. Cada ítem se puntúa de acuerdo al desempeño del menor con una calificación de 0,1, o 2 .

Para citar este artículo utilice la siguiente referencia: Jiménez, C.; Benavides, E. V.; Ornelas, M.; Rodríguez, J.M.; Lira, S.O.; Laguna, A. (2020). Habilidades motrices y su relación con las actividades y creencias parentales en preescolares; comparaciones por nivel socio-económico. Sportis Sci J, 6 (1), 122144. DOI: https://doi.org/10.17979/sportis.2020.6.1.5388 
Artículo Original. Habilidades motrices y su relación con las actividades y creencias parentales en preescolares; comparaciones por nivel socio-económico. Vol. 6, nº. 1; p. 122-144, enero 2020. A Coruña. España ISSN 2386-8333

Cuestionario para padres de familia. El cuestionario fue desarrollado específicamente para esta investigación, consta de dos secciones; la primera incluye 11 preguntas sobre las creencias de los padres con respecto al desarrollo de las habilidades de motricidad fina y gruesa en la etapa preescolar; los padres responden en una escala de tipo Likert que va desde "no importante" hasta "extremadamente importante." La segunda consta de un total de 20 ítems e indaga sobre la frecuencia con que los padres practican actividades de motricidad fina y gruesa con sus hijos; los padres responden en una escala tipo Likert que va desde "rara vez o nunca" hasta "la mayoría de los días de la semana." Los ítems se muestran en las Tablas 1 y 2 .
\end{abstract}

\title{
Análisis de Datos
}

Para analizar si existen diferencias en las creencias sobre el desarrollo motor y la frecuencia con que los padres realizan actividades motrices con sus hijos en NSE alto y bajo se utilizó análisis de varianza mixto; se utilizó Análisis Multivariado de Covarianza MANCOVA para analizar el desempeño de los niños en motricidad gruesa y fina. Por último, se analizó la relación entre creencias, frecuencia de actividades y desempeño de los niños con correlaciones bivariadas.

\section{Resultados}

La muestra consta de un total de 75 niños de preescolar, 39 de NSE bajo (17 mujeres; $M$ edad $=52.18$ meses, $S D=6.94$; rango $=36-66$ meses) y 36 menores de NSE alto $(16$ mujeres; $M$ edad $=51.05$ meses, $\mathrm{SD}=9.70 ;$ rango $=37-70$ meses $)$ la ciudad de Chihuahua.

No se encontró diferencia entre la media de edad de los 2 grupos $t(73)=0.57, p>.05, d=$ .13. Tampoco se encontró diferencia en cuanto a la proporción de hombres y mujeres entre los dos grupos, $\chi^{2}(1)=0.02, p>.05, V$ de Cramer $=.02$.

Los padres de NSE bajo reportaron un nivel educativo significativamente más bajo que el de los padres de NSE alto. El 80\% de los padres de NSE bajo reportaron un nivel máximo de estudios de secundaria o menos, en contraste con los padres de NSE alto de quienes el $80 \%$ reportaron haber concluido estudios universitarios, $\chi^{2}(6)=43.63, p<.001$, $V$ de Cramer $=.92$. Por su parte, el $70 \%$ de las madres de NSE bajo reportaron un grado

Para citar este artículo utilice la siguiente referencia: Jiménez, C.; Benavides, E. V.; Ornelas, M.; Rodríguez, J.M.; Lira, S.O.; Laguna, A. (2020). Habilidades motrices y su relación con las actividades y creencias parentales en preescolares; comparaciones por nivel socio-económico. Sportis Sci J, 6 (1), 122144. DOI: https://doi.org/10.17979/sportis.2020.6.1.5388 
Artículo Original. Habilidades motrices y su relación con las actividades y creencias parentales en preescolares; comparaciones por nivel socio-económico. Vol. 6, nº. 1; p. 122-144, enero 2020. A Coruña. España ISSN 2386-8333

máximo de estudios alcanzados de preparatoria o menor, mientras que el $80 \%$ de las madres de NSE alto reportaron haber obtenido un grado universitario, $\chi^{2}(4)=35.27, p<$ $.001, V$ de Cramer $=.80$.

Las Tablas 1 y 2 muestran las estadísticas descriptivas y la fiabilidad de los cuestionarios completados por los padres con respecto a la frecuencia con que realizan actividades de motricidad fina y gruesa con sus hijos y sobre las creencias acerca de la importancia que tienen diversos hitos del desarrollo motor grueso y fino. 

socio-económico. Vol. 6, nº. 1; p. 122-144, enero 2020. A Coruña. España ISSN 2386-8333

Tabla 1. Estadísticas descriptivas y fiabilidad para el cuestionario sobre la frecuencia de actividades de motricidad por NSE.

\begin{tabular}{|c|c|c|c|c|}
\hline \multirow[b]{3}{*}{ Motricidad Gruesa } & \multicolumn{2}{|c|}{ NSE bajo } & \multicolumn{2}{|c|}{ NSE alto } \\
\hline & $M$ & $S D$ & $M$ & $S D$ \\
\hline & 2.12 & 0.77 & 1.94 & 0.72 \\
\hline Fiabilidad & .80 & & .82 & \\
\hline $\begin{array}{l}\text { Le enseño a mí hijo a identificar el lado izquierdo y } \\
\text { derecho de su cuerpo }\end{array}$ & 2.28 & 1.47 & 2.31 & 1.24 \\
\hline Le enseño a mí hijo a trepar. & 2.00 & 1.47 & 1.97 & 1.48 \\
\hline $\begin{array}{l}\text { Animo a mí hijo a jugar en columpios, sube-y-bajas, } \\
\text { resbaladeros. }\end{array}$ & 2.62 & 1.23 & 2.66 & 0.89 \\
\hline Le enseño a mí hijo a andar en bicicleta o triciclo & 2.44 & 1.43 & 2.17 & 1.11 \\
\hline $\begin{array}{l}\text { Procuro que mi hijo realice actividades que le ayuden con } \\
\text { el equilibrio: saltar en un pie, caminar sobre una línea } \\
\text { recta. }\end{array}$ & 2.28 & 1.52 & 1.92 & 1.18 \\
\hline Le enseño a mí hijo a saltar la cuerda. & 1.21 & 1.42 & 0.64 & 0.99 \\
\hline $\begin{array}{l}\text { Animo a mí hijo a imitar diversas posturas, caminar como } \\
\text { animales diferentes. }\end{array}$ & 1.38 & 1.41 & 1.19 & 1.28 \\
\hline Animo a mí hijo a correr. & 3.35 & 0.87 & 3.05 & 0.92 \\
\hline $\begin{array}{l}\text { Mi hijo participa en actividades deportivas fuera de la } \\
\text { escuela. }\end{array}$ & 0.82 & 1.23 & 1.17 & 1.52 \\
\hline Animo a mí hijo a bailar al ritmo de la música. & 3.31 & 0.95 & 2.97 & 1.08 \\
\hline Animo a mí hijo a andar sobre las puntas de los pies. & 1.64 & 1.44 & 1.28 & 1.36 \\
\hline Motricidad Fina & 2.53 & $\mathbf{0 . 8 5}$ & 2.37 & 0.73 \\
\hline Fiabilidad & .78 & & .79 & \\
\hline Le enseño a mí hijo a tomar el lápiz correctamente & 2.87 & 1.24 & 2.92 & 1.08 \\
\hline Recortamos & 2.28 & 1.30 & 2.44 & 1.00 \\
\hline Jugamos con plastilina & 1.92 & 1.29 & 2.50 & 1.05 \\
\hline Dibujamos y/o pintamos & 3.10 & 0.97 & 3.19 & 0.71 \\
\hline $\begin{array}{l}\text { Le enseño a mí hijo(a) a abrir y cerrar envases con tapa } \\
\text { rosca. }\end{array}$ & 2.74 & 1.50 & 2.33 & 1.33 \\
\hline Le enseño a mi hijo a abotonarse la ropa & 3.00 & 1.34 & 2.50 & 1.32 \\
\hline Separamos objetos pequeños con las manos. & 2.03 & 1.51 & 2.05 & 1.28 \\
\hline Le enseño a mí hijo a abrocharse las agujetas & 2.28 & 1.59 & 1.03 & 1.30 \\
\hline Actividad fina y gruesa & 2.33 & $\mathbf{0 . 7 5}$ & 2.16 & 0.67 \\
\hline Fiabilidad Global & .88 & & .88 & \\
\hline
\end{tabular}

Nota. La fiabilidad es alfa de Cronbach. Los padres respondían a la pregunta: Por favor indique qué tan seguido realiza las siguientes actividades con su hijo(a) en una escala likert: $0=$ rara vez o nunca, $1=$ una vez al mes, $2=$ una vez a la semana, $3=$ varios dias a la semana, 4 = la mayoría de los días de la semana.

Para citar este artículo utilice la siguiente referencia: Jiménez, C.; Benavides, E. V.; Ornelas, M.; Rodríguez, J.M.; Lira, S.O.; Laguna, A. (2020). Habilidades motrices y su relación con las actividades y creencias parentales en preescolares; comparaciones por nivel socio-económico. Sportis Sci J, 6 (1), 122144. DOI: https://doi.org/10.17979/sportis.2020.6.1.5388 
Artículo Original. Habilidades motrices y su relación con las actividades y creencias parentales en preescolares; comparaciones por nivel socio-económico. Vol. 6, nº. 1; p. 122-144, enero 2020. A Coruña. España ISSN 2386-8333

Tabla 2. Estadísticas descriptivas y fiabilidad para cuestionario sobre creencias de motricidad por NSE.

\begin{tabular}{|c|c|c|c|c|}
\hline & \multicolumn{2}{|c|}{ NSE bajo } & \multicolumn{2}{|c|}{ NSE alto } \\
\hline & $M$ & $S D$ & $M$ & $S D$ \\
\hline Motricidad Gruesa & 2.50 & 0.94 & 2.87 & 0.83 \\
\hline Fiabilidad & .86 & & .88 & \\
\hline Pararse en un pie sin apoyo. & 2.25 & 1.18 & 2.53 & 1.08 \\
\hline $\begin{array}{l}\text { Saltar hacia adelante con los } \\
\text { pies juntos. }\end{array}$ & 3.03 & 1.19 & 2.78 & 1.05 \\
\hline $\begin{array}{l}\text { Subir escaleras alternando los } \\
\text { pies. }\end{array}$ & 2.58 & 1.05 & 2.89 & 1.09 \\
\hline $\begin{array}{l}\text { Caminar sobre una línea } \\
\text { marcada en el suelo. }\end{array}$ & 2.35 & 1.23 & 2.75 & 1.08 \\
\hline Identificar izquierda y derecha. & 2.22 & 1.32 & 3.39 & 0.73 \\
\hline Motricidad Fina & 2.80 & 0.81 & 2.97 & 0.68 \\
\hline Fiabilidad & .86 & & .89 & \\
\hline Desabotonar y desatar agujetas. & 2.50 & 0.81 & 2.52 & 0.84 \\
\hline Dibujar figuras geométricas. & 2.84 & 1.07 & 2.86 & 0.93 \\
\hline Recortar. & 2.89 & 0.98 & 3.22 & 0.83 \\
\hline $\begin{array}{l}\text { Comer con tenedor o cuchara } \\
\text { (sin ayuda). }\end{array}$ & 3.11 & 1.07 & 3.50 & 0.65 \\
\hline $\begin{array}{l}\text { Agarrar con el dedo índice y el } \\
\text { pulgar. }\end{array}$ & 2.41 & 1.14 & 3.06 & 0.98 \\
\hline Abrocharse las agujetas. & 2.84 & 1.21 & 2.66 & 0.86 \\
\hline Creencias motricidad global & 2.53 & $\mathbf{0 . 8 5}$ & 2.92 & 0.72 \\
\hline Fiabilidad Global & .93 & & .93 & \\
\hline
\end{tabular}

Nota. La fiabilidad es alfa de Cronbach. Los padres respondían a la pregunta: Por favor indique qué tan importante es para usted que su hijo (a) sea capaz de realizar las siguientes actividades antes de ingresar a primero de primaria en una escala Likert: $0=$ no importante, 1 = ni importante ni no importante, $2=$ importante, $3=$ muy importante, $4=$ extremadamente importante. 
Artículo Original. Habilidades motrices y su relación con las actividades y creencias parentales en preescolares; comparaciones por nivel socio-económico. Vol. 6, nº. 1; p. 122-144, enero 2020. A Coruña. España ISSN 2386-8333

\section{Frecuencia de actividades de motricidad gruesa y fina que reportan los padres de NSE}

\section{alto y bajo.}

Para determinar si existe diferencia significativa entre la frecuencia de actividades de motricidad fina y gruesa que reportan los padres por nivel socio-económico, se realizó un Análisis de Varianza Mixto de 2 (NSE alto y bajo) x 2 (actividades de motricidad gruesa y fina) (ver Tabla 3). Los resultados indican que los padres de ambos NSE reportan realizar actividades de motricidad fina con mayor frecuencia que actividades de motricidad gruesa (ver Figura 1).

Tabla 3. Estadística inferencial de los análisis de frecuencia de actividades motrices.

\begin{tabular}{lcl}
\hline & $F(1,73)$ & $\eta_{p}^{2}$ \\
\hline Nivel socio-económico & 1.05 & .03 \\
Actividades motricidad gruesa y fina & $38.91 * * *$ & .35 \\
NSE x Actividades & 0.03 & .00 \\
\hline
\end{tabular}

$* * * p<.001$

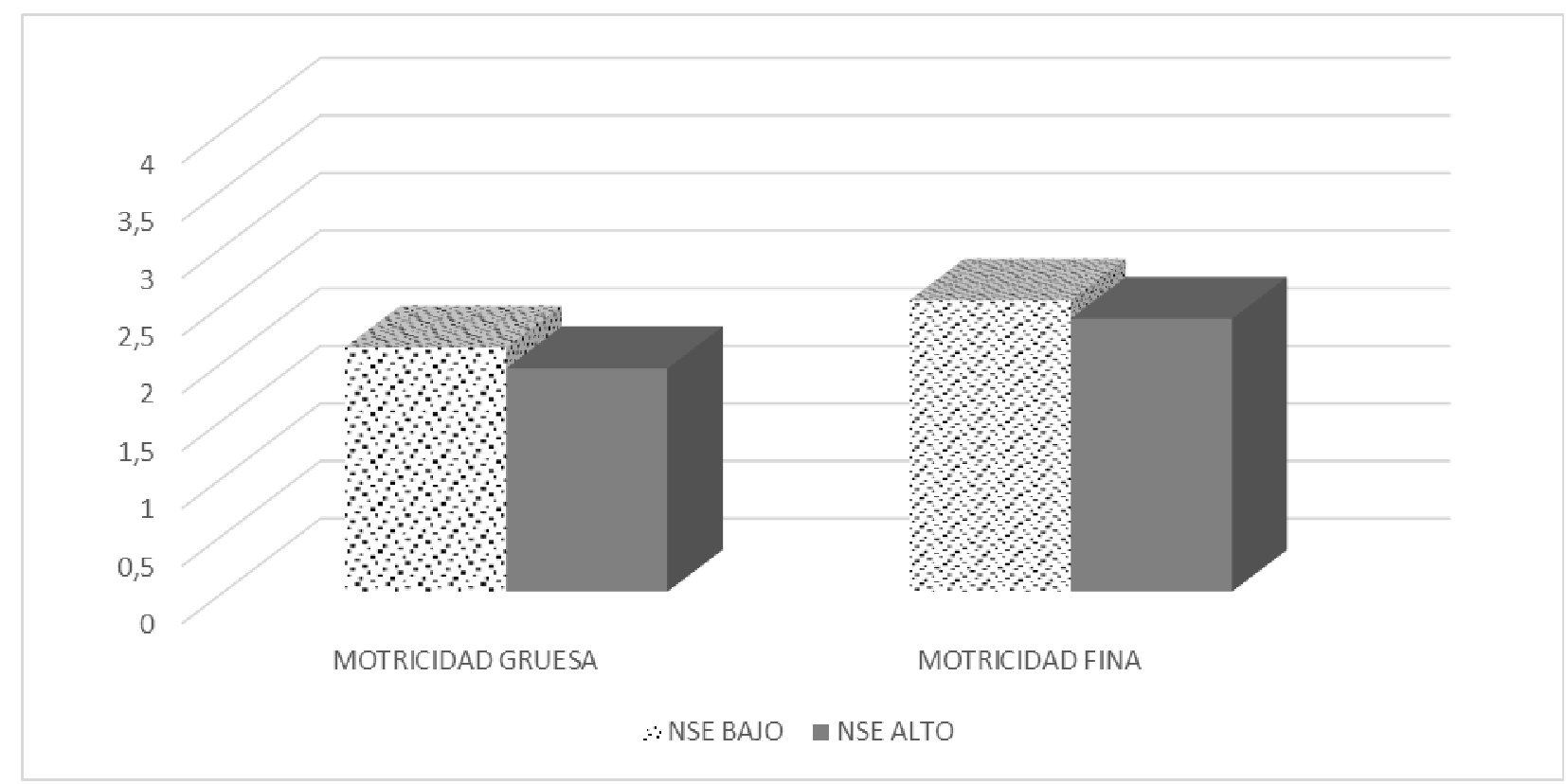

Figura 1. Resultados de frecuencia de actividades motrices por nivel socio-económico 
Artículo Original. Habilidades motrices y su relación con las actividades y creencias parentales en preescolares; comparaciones por nivel socio-económico. Vol. 6, nº. 1; p. 122-144, enero 2020. A Coruña. España ISSN 2386-8333

\section{Creencias de los padres de NSE alto y bajo con respecto al desarrollo de las habilidades motrices gruesa y fina.}

Para determinar si existe diferencia significativa entre las creencias de los padres de NSE alto y bajo con respecto a la importancia del desarrollo de los hitos de motricidad gruesa y fina, se realizó un Análisis de Varianza Mixto de 2 (NSE alto y bajo) x 2 (creencias gruesa y fina) los resultados (Tabla 4) indican que los padres de ambos NSE, le conceden mayor importancia al desarrollo de la motricidad fina que a la motricidad gruesa (Figura 2).

Tabla 4. Estadística inferencial de los análisis de creencias con respecto al desarrollo de las habilidades motrices.

\begin{tabular}{lcl}
\hline & $F(1,67)$ & $\eta_{p}^{2}$ \\
\hline Nivel socio-económico & 1.75 & .03 \\
Creencias motricidad gruesa y fina & $10.36^{* *}$ & .13 \\
NSE x Creencias & 1.98 & .03 \\
\hline
\end{tabular}
$* * p<.01$

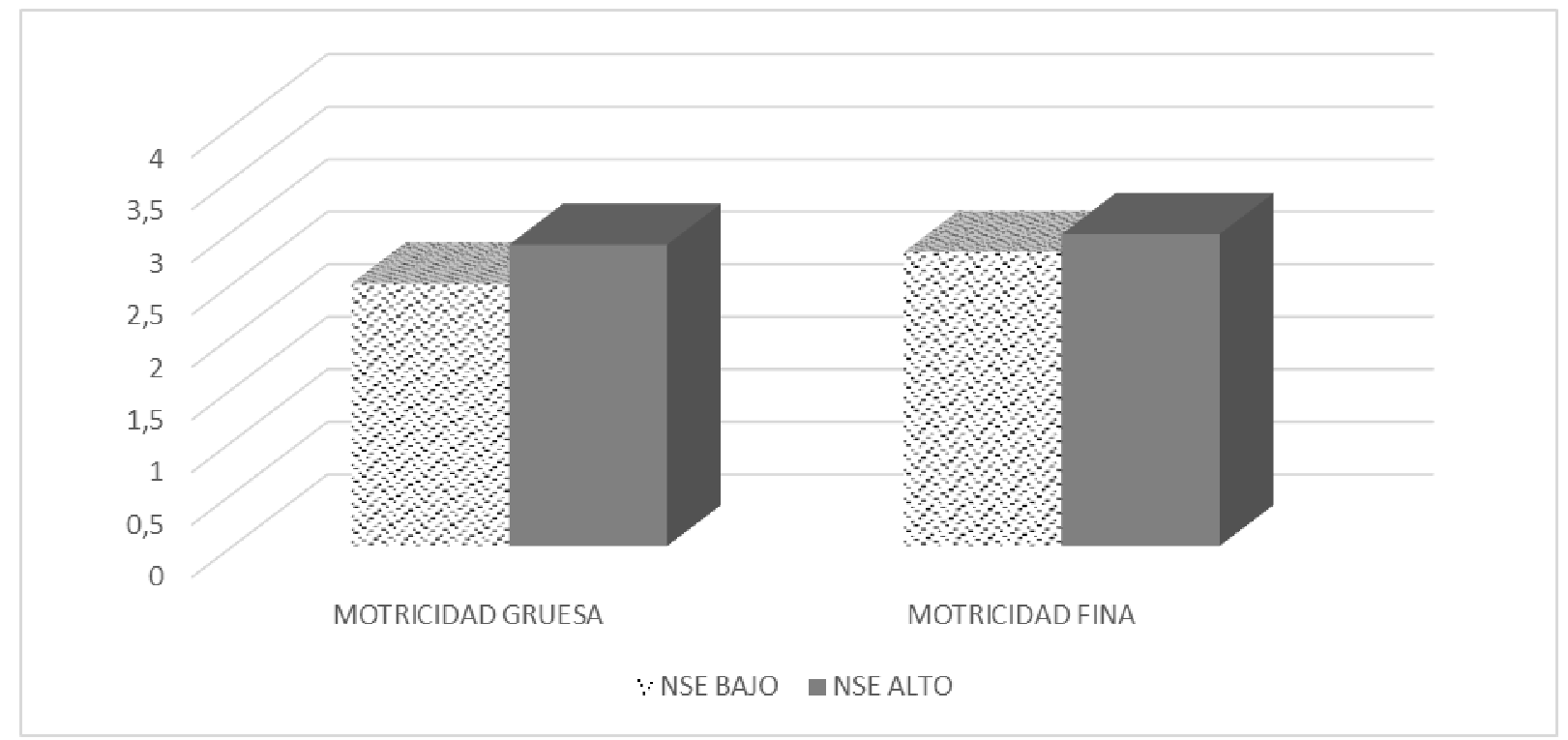

Figura 2. Creencias con respecto al desarrollo motor parentales por NSE. 


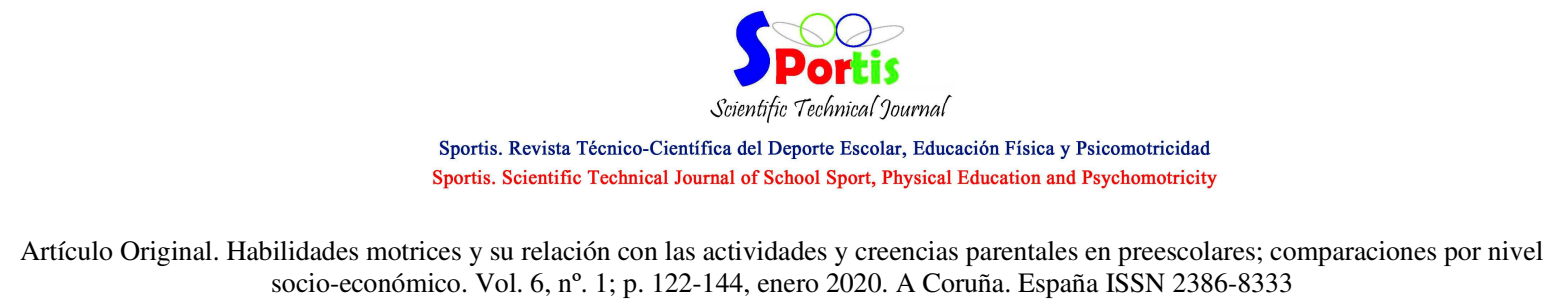
socio-económico. Vol. 6, nº 1; p. 122-144, enero 2020. A Coruña. España ISSN 2386-8333

\section{Habilidades motrices gruesa y fina entre preescolares de NSE alto y bajo.}

La Tabla 5 muestra las medias alcanzadas por los preescolares en las sub-escalas de motricidad gruesa y fina del Inventario de Desarrollo Battelle y la fiabilidad obtenida para cada sub-escala por NSE.

Para analizar si existen diferencias en el desempeño de los niños en motricidad fina y gruesa por NSE, se realizó un análisis multivariado de covarianza ${ }^{1}$ con nivel socio-económico como factor inter-sujetos y la variable edad como covariable; el análisis global fue seguido por ANOVAs univariadas.

Los resultados globales indican que existen diferencias significativas en el desempeño por nivel socio-económico, $F(2,63)=15.64, p=.000, \eta_{p}^{2}=.33$; el efecto de la edad fue significativo $F(2,63)=25.79, p=.000, \eta_{p}^{2}=.45$. Los resultados de las ANOVAs univariadas indican que los niños de NSE bajo obtuvieron una puntuación significativamente más alta en motricidad gruesa que sus pares de NSE alto, $F(1,64)=29.85, p=.000, \eta_{p}^{2}=.32$; sin embargo, no se encontraron diferencias significativas entre las puntuaciones de motricidad fina de ambos grupos (NSE alto y bajo), $F(1,64)=3.37, p=.071, \eta_{p}^{2}=.05$.

Tabla 5. Resultados de sub-escalas de motricidad gruesa y fina por NSE.

\begin{tabular}{lcccc}
\hline Inventario de & Desarrollo & NSE bajo & \multicolumn{3}{c}{ NSE alto } \\
\cline { 2 - 5 } Battelle & $M$ & $S D$ & $M$ & $S D$ \\
\hline Motricidad Gruesa & 81.76 & 5.03 & 75.58 & 6.52 \\
Fiabilidad & .82 & & .86 & \\
Motricidad Fina & 48.00 & 7.76 & 43.79 & 8.28 \\
Fiabilidad & .88 & & .90 & \\
\hline
\end{tabular}

Nota. La fiabilidad es alfa de Cronbach.

${ }^{1}$ Se utilizó el método percentil bootstrap para minimizar el sesgo por a) falta de normalidad de las variables y b) el tamaño de la muestra (Vallejo, Cuesta, Fernández y Herrero, 2006; Vallejo, Fernández y Livacic-Rojas, 2010).

Para citar este artículo utilice la siguiente referencia: Jiménez, C.; Benavides, E. V.; Ornelas, M.; Rodríguez, J.M.; Lira, S.O.; Laguna, A. (2020). Habilidades motrices y su relación con las actividades y creencias parentales en preescolares; comparaciones por nivel socio-económico. Sportis Sci J, 6 (1), 122144. DOI: https://doi.org/10.17979/sportis.2020.6.1.5388 


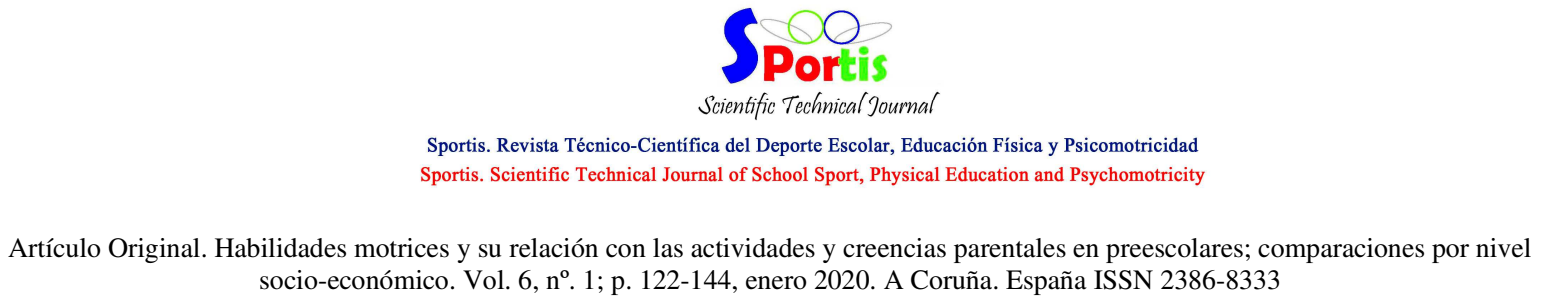
socio-económico. Vol. 6, nº 1; p. 122-144, enero 2020. A Coruña. España ISSN 2386-8333

\section{Relación entre las creencias y frecuencia de actividades motrices reportada por los padres se relacionan y las habilidades motrices gruesa y fina obtenidas por sus hijos.}

Para analizar si existe una relación entre la frecuencia de actividades motrices, creencias de los padres con respecto al desarrollo motor y las habilidades de motricidad gruesa y fina de los preescolares, se analizaron las correlaciones bivariadas por nivel socioeconómico (ver Tabla 6).

En ningún grupo de NSE se encontró relación entre la frecuencia de actividades motrices reportadas por los padres y el desempeño de los niños; el desempeño de los niños tampoco se relacionó con las creencias de los padres. Sin embargo, la frecuencia con que los padres reportan realizar actividades de motricidad gruesa se relacionan positivamente con las creencias de los padres en ambos NSE.

En ambos NSE, los padres que reportaron realizar actividades de motricidad gruesa con mayor frecuencia, también realizan actividades de motricidad fina; así mismo, se encontró una correlación significativa en cuanto la creencia sobre la importancia del desarrollo de la motricidad gruesa y fina.

Tabla 6. Correlaciones bivariadas NSE bajo $(n=31-39)$ debajo de la diagonal; NSE Alto $(n=$ 34 - 36) arriba de la diagonal.

\begin{tabular}{lccccccc}
\hline & $\begin{array}{c}\text { Battelle } \\
\text { MG }\end{array}$ & $\begin{array}{c}\text { Battelle } \\
\text { MF }\end{array}$ & $\begin{array}{c}\text { Activ. } \\
\text { MG }\end{array}$ & $\begin{array}{c}\text { Activ. } \\
\text { MF }\end{array}$ & $\begin{array}{c}\text { Creencias } \\
\text { MG }\end{array}$ & $\begin{array}{c}\text { Creencias } \\
\text { MF }\end{array}$ & Edad \\
\hline Battelle MG & - & $.71^{* *}$ & -.13 & -.01 & .05 & .07 & $.69 * *$ \\
Battelle MF & $.56^{* *}$ & - & -.19 & -.15 & -.07 & -.11 & $.43^{*}$ \\
Act. MG & .13 & .23 & - & $.71^{* *}$ & $.39 *$ & .14 & -.09 \\
Act. MF & .19 & .30 & $.72^{* *}$ & - & .14 & .03 & .12 \\
Creencias MG & -.17 & -.11 & $.36^{* *}$ & .18 & - & $.79 * *$ & -.04 \\
Creencias MF & -.16 & -.06 & .22 & .13 & $.88 * *$ & - & .04 \\
Edad & $.68^{* *}$ & $.43 * *$ & .25 & .28 & .08 & -.04 & - \\
\hline
\end{tabular}

Nota: $\mathrm{MG}=$ motricidad gruesa, $\mathrm{MF}=$ motricidad fina. ${ }^{*} p<.05, * * p<.01$

Para citar este artículo utilice la siguiente referencia: Jiménez, C.; Benavides, E. V.; Ornelas, M.; Rodríguez, J.M.; Lira, S.O.; Laguna, A. (2020). Habilidades motrices y su relación con las actividades y creencias parentales en preescolares; comparaciones por nivel socio-económico. Sportis Sci J, 6 (1), 122144. DOI: https://doi.org/10.17979/sportis.2020.6.1.5388 


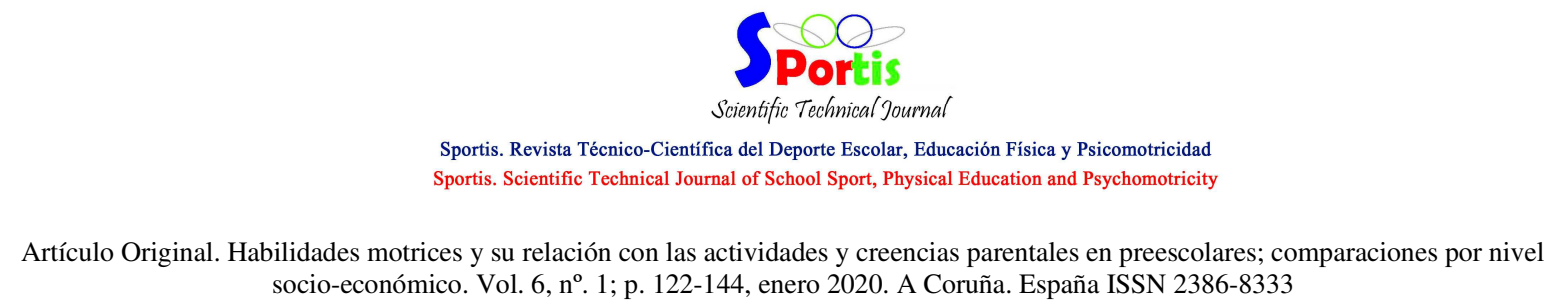
socio-económico. Vol. 6, nº 1; p. 122-144, enero 2020. A Coruña. España ISSN 2386-8333

\section{Discusión}

El presente estudio tenía cuatro objetivos principales analizar si existe diferencia en: a) la frecuencia de actividades de motricidad gruesa y fina que reportan entre los padres de NSE alto y bajo; b) las creencias de los padres de NSE alto y bajo con respecto al desarrollo de las habilidades motrices c) las habilidades de motricidad gruesa, y motricidad fina entre preescolares de NSE alto y bajo; y si las creencias y frecuencia de actividades motrices reportada por los padres se relacionan con las habilidades motrices gruesa y fina obtenidas por sus hijos.

Encontramos que los padres de ambos NSE realizan con mayor frecuencia en el hogar y le dan mayor importancia al desarrollo de las habilidades de motricidad fina en comparación con la gruesa .. Considerando que los menores evaluados asistían a preescolar, nuestros resultados pueden estar relacionados con el tipo de actividades y tareas que se enfatizan en el ambiente escolar, por ejemplo, las maestras pueden encargar a los niños tareas que incluyan actividades de motricidad fina como recortar y dibujar, por ende, los padres de familia reporten realizar este tipo de actividades con mayor frecuencia; sin embargo al observar que las correlaciones entre creencias de motricidad fina y frecuencia reportada de actividades de motricidad fina en ambos NSE no fueron significativas, lo que implica que es necesario mayor investigación sobre la relación de estas variables y también así para conocer el impacto de las tareas encargadas por las educadoras de centros preescolares sobre la frecuencia y tipo de actividades motrices implementadas por los padres de familia (p.ej., Smith, Robbins, Stagman, y Mathur, 2013).

Así mismo, otra razón por la cual se reporten actividades de motricidad fina con mayor frecuencia que actividades de motricidad gruesa puede ser, como afirma Coronado (2011), que el ambiente o espacio no siempre es el adecuado para que se desarrollen las habilidades motrices gruesas, ya que los hogares no suelen tener espacios amplios y salir a la calle a jugar no es viable por razones de seguridad, o bien un parque pudiese ser el espacio más idóneo pero requiere una mayor inversión de tiempo.

Para citar este artículo utilice la siguiente referencia: Jiménez, C.; Benavides, E. V.; Ornelas, M.; Rodríguez, J.M.; Lira, S.O.; Laguna, A. (2020). Habilidades motrices y su relación con las actividades y creencias parentales en preescolares; comparaciones por nivel socio-económico. Sportis Sci J, 6 (1), 122144. DOI: https://doi.org/10.17979/sportis.2020.6.1.5388

http://revistas.udc.es/ 


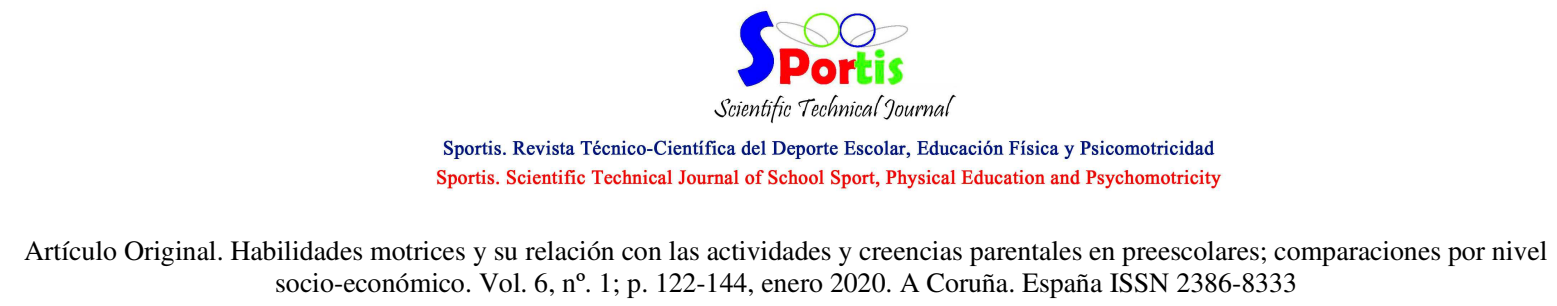

Con respecto a las actividades de motricidad gruesa, nuestros hallazgos indican que la frecuencia con que los padres reportan realizar este tipo de actividades correlaciona positivamente con las creencias sobre la importancia del desarrollo de habilidades de motricidad gruesa, de manera que aquellos padres que consideran importante el desarrollo de las habilidades de motricidad gruesa, son aquellos que reportan realizar actividades de éste tipo con mayor frecuencia y viceversa. Estos resultados cobran importancia sobre todo a la luz de los hallazgos por Lindsay, Wallington, Muñoz, y Greany, (2018) quienes refieren que es posible que algunos padres no se preocupen tanto por las actividades de motricidad gruesa porque no las relacionan directamente con el aprendizaje, sino más bien las asocian con el desarrollo deportivo, que comúnmente es percibido como algo secundario ó porque consideran otras actividades tales como los videojuegos o utilizar aparatos electrónicos como una forma igualmente favorable de convivencia. Los resultados de nuestro estudio pueden sentar la base para llevar a cabo estudios de intervención con los padres de familia con el objetivo de incrementar el conocimiento de los mismos con respecto a los beneficios de fomentar actividades de motricidad gruesa con sus hijos y observar el efecto en la frecuencia con que reportan realizar actividades de ésta naturaleza.

En el mismo sentido, Gomes et al., encontraron una relación entre creencias y frecuencia de actividades tanto de motricidad gruesa como fina, en inconsistencia con nuestros resultados en los cuales solamente se encontró relación en el caso de las actividades de motricidad gruesa, posiblemente debido a el rango de edad estudiado, ya que los datos de Gomes et al fueron obtenidos en niños entre 12 y 24 meses, a diferencia de nuestros datos que provienen de niños de 52 meses, que se encuentran en otra etapa del desarrollo con demandas y dificultades propias del nivel preescolar como por ejemplo al sujetar el lápiz, o recortar, lo que hace que los padres de familia se enfoquen más en ello, mientras ocurre lo contrario con las habilidades de motricidad gruesa (correr, saltar) ya que a esa edad están adquiridas y únicamente aquellos padres que son conscientes de la importancia de continuar estimulando estas habilidades, las promueven con mayor frecuencia

En relación al desempeño de los niños, los participantes de NSE bajo obtuvieron una puntuación significativamente más alta en motricidad gruesa que sus pares de NSE alto; sin

Para citar este artículo utilice la siguiente referencia: Jiménez, C.; Benavides, E. V.; Ornelas, M.; Rodríguez, J.M.; Lira, S.O.; Laguna, A. (2020). Habilidades motrices y su relación con las actividades y creencias parentales en preescolares; comparaciones por nivel socio-económico. Sportis Sci J, 6 (1), 122144. DOI: https://doi.org/10.17979/sportis.2020.6.1.5388

http://revistas.udc.es/ 


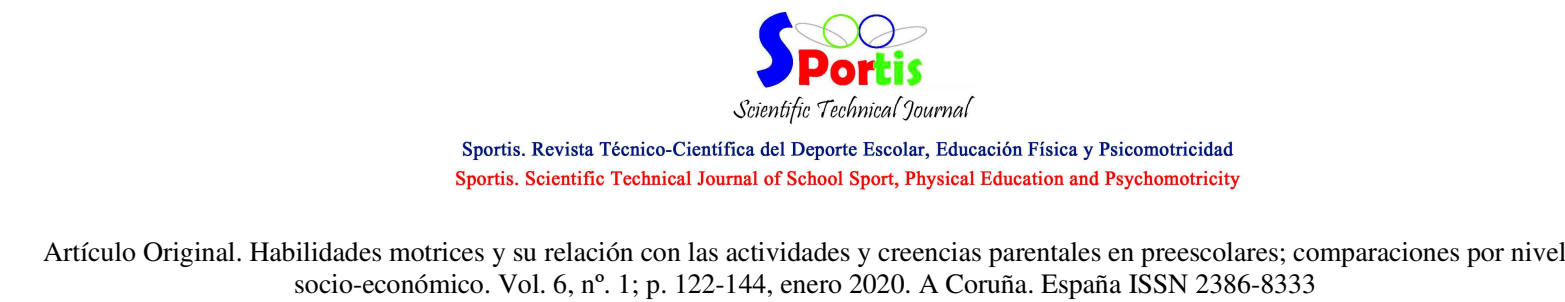

embargo no se encontraron diferencias significativas con respecto a las puntuaciones obtenidas en motricidad fina. Estos resultados difieren de los datos de estudios anteriores (Leyva y Valdés, 2016; Liu et al., 2015; Valdés Arriaga y Spencer Contreras, 2011) quienes encontraron que los niños de NSE alto obtenían puntuaciones más altas en pruebas de motricidad; los autores refieren que los menores pertenecientes a niveles socio-económicos mas bajos carecen de estimulación adecuada lo cual repercute en el desarrollo psicomotor; en contraste los hallazgos de Farkas y Corthorn (2012) afirman que padres más informados, preocupados y que estimulan e interactúan mas con sus hijos, tenderían a contrarrestar los efectos del NSE y promoverían un mejor desarrollo en sus hijos; en su estudio Wilk, Clark, Maltby, Smith, Tucker, y Gililand (2018) señalan que la escolaridad también es un factor importante, ya que los padres con un nivel máximo de estudios de preparatoria involucran mas a sus hijos en actividades motrices que aquellos con menos educación.

En esta investigación no se encontró relación entre la frecuencia de actividades motrices reportadas por los padres y el desempeño de los niños. Estudios previos (p.ej., Osorio et al., 2010) resaltan una relación significativa entre estimulación en el hogar y puntajes obtenidos por niños en pruebas de motricidad, sin embargo, utilizaron el inventario HOME como medida global de estimulación en el hogar, que difiere en gran medida del cuestionario empleado en ésta investigación. Existe la posibilidad de que las habilidades motrices estén más relacionadas con estimulación global constante (Osorio et al., 2010), y no con la frecuencia reportada por los padres como tampoco se relacionó con las creencias de los padres. Es necesario realizar más investigación para determinar si existe variabilidad en cuanto a las edades en que el desarrollo de las habilidades motrices son más sensibles al aumento de la estimulación en el hogar.

\section{Conclusiones}

El presente estudio integró cuatro variables que han sido consideradas por separado en la literatura: creencias parentales sobre el desarrollo motor, frecuencia de actividades motrices reportadas por los padres, desempeño motor de los niños y nivel socio-económico. Se encontró que las creencias sobre el desarrollo motor de los niños se relacionan con la

Para citar este artículo utilice la siguiente referencia: Jiménez, C.; Benavides, E. V.; Ornelas, M.; Rodríguez, J.M.; Lira, S.O.; Laguna, A. (2020). Habilidades motrices y su relación con las actividades y creencias parentales en preescolares; comparaciones por nivel socio-económico. Sportis Sci J, 6 (1), 122144. DOI: https://doi.org/10.17979/sportis.2020.6.1.5388

http://revistas.udc.es/ 

socio-económico. Vol. 6, nº. 1; p. 122-144, enero 2020. A Coruña. España ISSN 2386-8333

frecuencia con que se realizan actividades de ésta naturaleza, y que éstas variables no difieren por nivel socio-económico. Ni las creencias, ni actividades se relacionaron con el desempeño de los niños. El desempeño en motricidad gruesa sin embargo, sí tuvo variación por nivel socio-económico, siendo mejor en el grupo menos aventajado. Sin embargo,. Estos resultados sugieren que es necesario indagar más en el tema de las creencias y las actividades que realizan los padres y su relación con el desarrollo motor de sus hijos, así como estudiar factores distintos que pudiesen favorecer las habilidades motrices en preescolar (actividades escolares). Finalmente queda abierto como una futura línea de investigación a seguir, la influencia en el desarrollo motor de las prácticas de crianza (Grijalva, 2015), las cuales pueden diferir por nivel socioeconómico y/o el ambiente en el que se encuentran los infantes (Osorio et al., 2017). Es importante reconocer como limitación el tamaño de la muestra del presente estudio por lo cual es importante que se replique con un mayor número de participantes. 


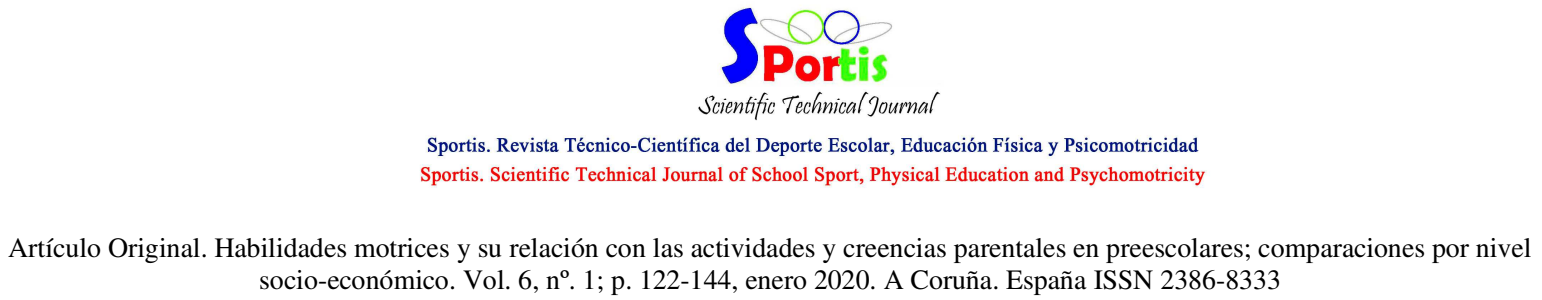
socio-económico. Vol. 6, nº. 1; p. 122-144, enero 2020. A Coruña. España ISSN 2386-8333

\section{Referencias Bibliográficas}

Abbot, A.L., Bartlett, D.J., Kneale Fanning, J.E., Kramer, J. (2000). Infant motor development and aspects of the home environment. Pediatric Physical Therapy, (12), 62-67. https://doi.org/10.1097/00001577-200012020-00003

Arufe-Giráldez, V. (2020). ¿Cómo debe ser el trabajo de educación física en educación infantil? (¿How should Physical Education work in Early Childhood Education be?). Retos, [S.1.], n. 37, p. 588-596, ISSN 1988-2041. Disponible en: https://recyt.fecyt.es/index.php/retos/article/view/74177

Berruezo y Adelantado, P.P. (2000). El contenido de la psicomotricidad. En Bottini, P. Psicomotricidad: prácticas y conceptos. 43-99. Madrid, España.

Bustamante Valdivia, A., Caballero Cartagena, L., Enciso Sarria, N., Salazar Távara, I., Teixeira e Seabra, A.F., Garganta de Silva, R.M., y Ribeiro Maia, J.A. (2008). Coordinación motora: Influencia de la edad, sexo, estatus socio-económico y niveles de adiposidad en niños Peruanos. Revista Brasileira de Cineantropometría \& Desempenho Humano, (10) 25-34. https://doi.org/10.5007/1980-0037.2008v10n1p25

Castillo, E. (2013). Cinco beneficios del juego en los niños. Recuperado de http://www.estampas.com/cuerpo-y-mente/120125

Coronado, R. (2011). Influencia de la estimulación ambiental del hogar, en las habilidades motoras gruesas de preescolares pertenecientes a las comunidades rurales de Puaucho y Bahía Mansa. Revista Horizonte Ciencias de la Actividad Física, 2(1), 28-43.

Díaz AA, Bacallao Gallestey J, Vargas-Machuca R, Aguilar Velarde R. (2017). Desarrollo infantil en zonas pobres de Perú. Revista Panamericana de Salud Pública., 41:e71 http://www.who.int/iris/handle/10665/309436.

Farkas, C., y Corthorn, C. (2012). Modelo explicativo del desarrollo temprano cognitivo, motor y de lenguaje en infantes chilenos de nivel socioeconómico bajo. Estudios de Psicología, 33(3), 311-323. https://doi.org/10.1174/021093912803758237

Para citar este artículo utilice la siguiente referencia: Jiménez, C.; Benavides, E. V.; Ornelas, M.; Rodríguez, J.M.; Lira, S.O.; Laguna, A. (2020). Habilidades motrices y su relación con las actividades y creencias parentales en preescolares; comparaciones por nivel socio-económico. Sportis Sci J, 6 (1), 122144. DOI: https://doi.org/10.17979/sportis.2020.6.1.5388 http://revistas.udc.es/ 

socio-económico. Vol. 6, nº. 1; p. 122-144, enero 2020. A Coruña. España ISSN 2386-8333

Freitas, T.C.B., Gabbard, C., Caçola, P., Montebelo M.I. L., y Santos, D.C.C. (2013). Family socio-economic status and the provision of motor affordances in the home. Brazilian Journal of Physical Therapy, (4)17, 319-327. https://doi.org/10.1590/S1413$\underline{35552013005000096}$

Gomes A.M., Fonseca Ribeiro, R., Vinolas Prat, B., de Castro Magalhães, de Souza Morais, R.S. (2017). Parental practices and beliefs on motor development in the first year of life.

(4)30, 769-779.

https://doi.org/10.1590/1980-5918.030.004.ao12

Grijalva A. (2015). Estilos de Crianza de los padres y su relación con el Desarrollo Psicomotriz en la Primera Infancia. Tesis de Grado. http://repositorio.pucesa.edu.ec/bitstream/123456789/1258/1/75841.pdf.

Leiva, M., Valdés, M. (2016). Modelo estadístico para predecir el puntaje de desarrollo psicomotor de 4 a 5 años de edad en función del nivel socioeconómico. Archivos de Pediatría de Uruguay, (87)1, 22-27.

Lindsay A.C., Wallington, S.F., Muñoz, M.A., y Greaney, M.L. (2018). A qualitative study conducted in the USA exploring Latino fathers'beliefs, attitudes and practices related to their young children'seating, physical activity and sedentary behaviours. Public Health and Nutrition, 21(2), 403-415. https://doi.org/10.1017/S1368980017002579

Liu, T., Hoffmann, C., y Hamilton, M. (2015). Motor skill performance by low SES and Typically developing children on the PDSM-2. Early Childhood Education Journal. $X X . h$ ttps://doi.org/10.1007/s10643-015-0755-9

Miquelote, A.F., Santos, D.C.C., Caçola, P.M., Montebelo M.I.L., y Gabbard, C. (2012). Infant Behaviour and Development, (35), 329-334. https://doi.org/10.1016/j.infbeh.2012.02.002

Miranda Yero, D., Jeffers Duarte, B., y Rey Benguría, C. (2019). El juego motriz en los preescolares. Opuntia Brava, 11(3), 188-198. https://doi.org/10.35195/ob.v11i3.803

Newborg, J. (2005). Batelle Developmental Inventory- Second Edition. Itasca, IL:Riverside. https://doi.org/10.1177/0734282907300382

Para citar este artículo utilice la siguiente referencia: Jiménez, C.; Benavides, E. V.; Ornelas, M.; Rodríguez, J.M.; Lira, S.O.; Laguna, A. (2020). Habilidades motrices y su relación con las actividades y creencias parentales en preescolares; comparaciones por nivel socio-económico. Sportis Sci J, 6 (1), 122144. DOI: https://doi.org/10.17979/sportis.2020.6.1.5388 

socio-económico. Vol. 6, nº. 1; p. 122-144, enero 2020. A Coruña. España ISSN 2386-8333

Osorio, E., Torres Sánchez, L., Hernández, M.C., López Carrillo, L., y Schnaas, L. (2010). Estimulación en el hogar y desarrollo motor en niños mexicanos de 36 meses. Salud $\begin{array}{llll}\text { Pública de } \quad \text { México, } & \text { (52)1, }\end{array}$ https://doi.org/10.1590/S0036-36342010000100004

Osorio, T. M.; Cortés, N.; Herrera, E. V; Orozco, L. A. (2017). Pautas de crianza y desarrollo psicomotor: una investigación en la primera infancia. Infancias Imágenes, 16(2), 242256.

Recart Herrera, M.I., Mathiesen Di Gregori, M.E., Herrera Garbarini, M.O. (2005). Relaciones entre algunas características de la familia del preescolar y su desempeño escolar posterior. Revista Enfoques Educacionales, (1)7, 105-123.

Rivera González, R., Méndez Ramírez, I., Figueroa Olea, M., Ávila Rosas, H., Sierra Cedillo, A., y Soler Limón, K. (2015). Relación entre ambiente, interacción, salud mental materna, y el desarrollo psicomotor del niño mediante análisis de ecuaciones estructurales. Investigación y Práctica en Psicología del Desarrollo(1),39-46 https://doi.org/10.33064/ippd1629

Rizzoli Córdoba, A., Martell Valdéz, L., Delgado Ginebra, I., Villasís Keever, M.A., Reyes Morales, H., O'Shea Cuevas, G., et al., (2015). Escrutinio poblacional del nivel de desarrollo infantil en menores de 5 años beneficiarios de PROSPERA en México.

Boletín Médico del Hospital Infantil de México, (72)6, 409-419. https://doi.org/10.1016/j.bmhimx.2015.10.003

Rodrigues, L.P., Saraiva, L., y Gabbard, C., (2005). Development and construct validation of an inventory for assessing the home environment for motor development. Research Quarterly for Excercise and Sport, (76) 2, 140-148. https://doi.org/10.1080/02701367.2005.10599276

Rodríguez Garcés, C., y Muñoz Soto, J. (2017). Rezago en el desarrollo infantil: la importancia de la calidad educativa del ambiente en el hogar. Revista Int. de Investigación en Ciencias Sociales. (13)2, 235-270. https://doi.org/10.18004/riics.2017.diciembre.253-270

Para citar este artículo utilice la siguiente referencia: Jiménez, C.; Benavides, E. V.; Ornelas, M.; Rodríguez, J.M.; Lira, S.O.; Laguna, A. (2020). Habilidades motrices y su relación con las actividades y creencias parentales en preescolares; comparaciones por nivel socio-económico. Sportis Sci J, 6 (1), 122144. DOI: https://doi.org/10.17979/sportis.2020.6.1.5388 
Silva, S.D., Flôres, F.S., Corrêa, S.L., Cordovil, R., y Copetti, F. (2017). Perceptual and Motor Skills, (124)1, $72-85$. https://doi.org/10.1177/0031512516676203

Smith, S., Robbins, T. A., Stagman, S. M., y Mathur, D. (2013). Parent engagement from preschool through grade 3: A guide for policymakers.

Valdés Arriagada, M. y Spencer Contreras, R. (2011). Influencia del nivel socioeconómico familiar sobre el desarrollo psicomotor de niños y niñas de 4 a 5 años de edad de la ciudad de Talca, Chile. Theoria, (20)2, 29-43.

Vallejo, G., Cuesta, M., Fernández, P., y Herrero, J. (2006). A comparison of the bootstrap-F, improved general approximation and Brown-Forsythe multivariate approaches in a mixed repeated measures design. Educational and Psychological Measurement, 66, $35-62$.

\section{https://doi.org/10.1177/0013164404273943}

Vallejo, G., Fernández, M. P., Tuero, E., y Rojas, P. E. L. (2010). Análisis de medidas repetidas usando métodos de remuestreo. Anales de Psicología/Annals of Psychology, 26(2), 400-409.

Wilk, P., Clark, A. F., Maltby, A., Smith, C., Tucker, P., y Gilliland, J. A. (2018). Examining individual, interpersonal, and environmental influences on children's physical activity levels. SSM-population health, 4 , 76-85. https://doi.org/10.1016/j.ssmph.2017.11.004

Zapata, B, y Rastrepo, J. (2013). Aprendizajes relevantes para los niños y las niñas en la primera infancia. Revista latinoamericana de ciencias sociales, niñez y juventud, 11 (1), pp. 217-227.

Para citar este artículo utilice la siguiente referencia: Jiménez, C.; Benavides, E. V.; Ornelas, M.; Rodríguez, J.M.; Lira, S.O.; Laguna, A. (2020). 